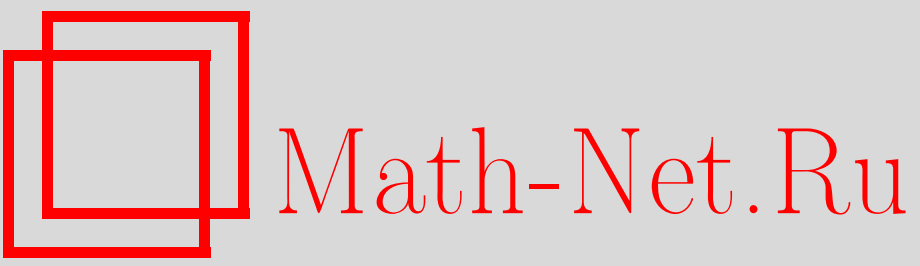

Г. Г. Ильюта, Субрезультанты Сильвестра, рациональные аппроксимации Коши, цепные дроби Тиле и высшие порядки Брюа, УМН, 2005, том 60, выпуск 2, 165166

DOI: https://doi.org/10.4213/rm1417

Использование Общероссийского математического портала Math-Net.Ru подразумевает, что вы прочитали и согласны с пользовательским соглашением http://www.mathnet.ru/rus/agreement

Параметры загрузки:

IP : 54.198 .55 .26

26 апреля 2023 г., 12:02:05 


\title{
СУБРЕЗУЛЬТАНТЫ СИЛЬВЕСТРА, РАЦИОНАЛЬНЫЕ АППРОКСИМАЦИИ КОШИ, ЦЕПНЫЕ ДРОБИ ТИЛЕ И ВЫСШИЕ ПОРЯДКИ БРЮА
}

\author{
Г. Г. ИльЮТА
}

Знаки разделенных разностей (р.p.) функции $f(y)$ в $(n+1)$-подмножествах множества $X_{\bar{m}}=$ $\left\{x_{1}<\cdots<x_{m}\right\} \subset \mathbb{R}$ определяют элемент высшего порядка Брюа (в.п.Б.) $B(m, n)$ [1]. В заметке этот факт обобщается для функций многих переменных. Мы опираемся на развитое А. Ласку и М.-П. Шютценберже исчисление элементарных (э.) p.p. $\partial_{i j}=(1-(i j)) /\left(x_{i}-x_{j}\right), \partial_{i, i+1}=\partial_{i}$, рассматриваемых как элементы групповой алгебры симметрической группы $S_{m}((i j)$ - транспозиция), действующей на функции перестановками переменных. Интерполяционньй ряд Ньютона $f(y)=\sum \partial_{i-1} \cdots \partial_{1}\left(f\left(x_{1}\right)\right)\left(y-x_{1}\right) \cdots\left(y-x_{i-1}\right)$ имеет обобщение для функций многих переменных [2], причем коэфффициентами являются примененные к функции композиции э.р.p. Сопоставление функции и каждой композиции э.р.р. элемента, соответствующего в.п.Б., можно рассматривать как комбинаторный вариант разложения функции в ряд Ньютона, и вопросом о сходимости такого ряда, по-видимому, следует считать обычный матроидньй вопрос о реализуемости. Мы ограничимся здесь рядом Ньютона для функции $f\left(y_{1}\right) \cdots f\left(y_{k}\right)$ и его коэффициентами, соответствующими симметризаторам Сильвестра $\left(\partial_{n-k} \cdots \partial_{1}\right) \cdots\left(\partial_{n-1} \cdots \partial_{k}\right)$ (они отвечают за невырожденность перечисленных в заглавии объектов). Аксиомы в определении в.п.Б. получены по следующей схеме: если соотношение для р.p. представлено в виде равной 0 суммы и не все слагаемые равны 0 , то среди них есть слагаемые разных знаков (получаем условие на индексы слагаемых, исключив непрерьвные параметры). Такие аксиомы обобщают определение перестановки как множества инверсий (для $B(m, n)$ см. [3]). В [4] обобщается определение перестановки как списка переставляемых символов. Мы обобщим определение $B(m, n)$, следуя подходу из [4]. В этом случае сопоставленньй функции элемент в.п.Б. можно интерпретировать как определяемьй линейной функцией порядок (по величине значений) на наборе точек. Близкая конструкция, в которой роль набора точек играет множество вершин многогранника матроида Кокстера, изучалась в [5]. Равенство $\partial_{m-1} \cdots \partial_{1}\left(x_{1}^{-1}\right)=(-1)^{m-1}\left(x_{1} \cdots x_{m}\right)^{-1}$ подсказьвает ариффметический аналог связи р.р. и в.п.Б.: для $A \in \mathbb{Z}, X_{\bar{m}} \subset \mathbb{N}$ циклический порядок на различных перестановках $s$ показателей $n_{1}, \ldots, n_{m} \in \mathbb{N} \cup 0$ определяют числа $A /\left(x_{1}^{s\left(n_{1}\right)} \cdots x_{m}^{s\left(n_{m}\right)}\right)^{-1}$ $\bmod \mathbb{Z}$.

При подходящих $m, k$ старшие коэффициенты в: 1$)$ интерполяционном многочлене для функции $\left.f\left(y_{1}\right) \cdots f\left(y_{k}\right)[6], 2\right)$ субрезультантах Сильвестра (остатках в алгоритме Евклида, примененном к $f(y)$ и $\left.\prod\left(y-x_{i}\right)\right)$ [7], 3) числителе и знаменателе аппроксимации Коши [8] (аппроксимации Ньютона-Паде [9]), 4) числителе и знаменателе дроби, полученной после сворачивания цепной дроби Тиле [10], имеют вид $d_{k}(\bar{m})=\operatorname{det}(f[i, j+m-k]), i, j=1, \ldots, k$, где $f[i, j]=$ $\partial_{j-1} \cdots \partial_{i}\left(f\left(x_{i}\right)\right)$ при $i \leqslant j$ и $f[i, j]=0$ при $i>j$. Обозначим через $C(m, n)$ множество $n$-подмножеств в $\bar{m}=\{1, \ldots, m\}$.

Теорема 1. 1) Ряд Ньютона для $\prod f\left(y_{i}\right)$ имеет вид (сумма по $0 \leqslant \lambda_{1} \leqslant \cdots \leqslant \lambda_{k}$ ) $f\left(y_{1}\right) \cdots f\left(y_{k}\right)=\sum \operatorname{det}\left(f\left[i, j+\lambda_{j}\right]\right) \operatorname{det}\left(\left(y-x_{1}\right) \cdots\left(y-x_{j+\lambda_{j}-1}\right)\right) / \prod\left(y_{i}-y_{j}\right)$.

2) $\operatorname{det}\left(f\left[i, j+\lambda_{j}\right]\right)=\left(\partial_{\lambda_{1}} \cdots \partial_{1}\right) \cdots\left(\partial_{k+\lambda_{k}-1} \cdots \partial_{k}\right)\left(f\left(x_{1}\right) \cdots f\left(x_{k}\right)\right)$.

3) $d_{k}(\bar{m}) d_{k-2}(\overline{m-1} \backslash 1)=d_{k-1}(\overline{m-1}) d_{k-1}(\bar{m} \backslash 1)-d_{k-1}(\overline{m-1} \backslash 1) d_{k-1}(\bar{m})$.

4) $d_{k}(\bar{m})=(-1)^{k} \sum_{i=1}^{k+1} d_{k}(\bar{m} \backslash i) / \prod_{i \neq j=1}^{k+1}\left(x_{i}-x_{j}\right)$.

5) $0=\sum_{i=1}^{k+2} d_{k}(\bar{m} \backslash i) / \prod_{i \neq j=1}^{k+2}\left(x_{i}-x_{j}\right)$.

В [11] определены в.п.Б. $B(m, n, k)$ как множество отображений $c: C(m, n+1) \rightarrow\{-1,1\}$, для которых все последовательности $c\left(I \backslash i_{1}\right), \ldots, c\left(I \backslash i_{n+2}\right), I=\left\{i_{1}<\cdots<i_{n+2}\right\} \in C(m, n+2)$, имеют не более $k$ перемен знака. Назовем в.п.Б. $B(m)$ подмножество элементов $\varepsilon=\left(\varepsilon_{n k}\right)$ прямого произведения всех $B(m, n, k)$ по $n$ и $k, n \geqslant k-1$, удовлетворяющих условиям: 1) если

Работа выполнена при поддержке Российского фонда фундаментальных исследований (грант № 04-01-00762). 
для $j_{1}<\cdots<j_{k+1} \in I$ последовательность $\varepsilon_{n k}\left(I \backslash j_{1}\right), \ldots, \varepsilon_{n k}\left(I \backslash j_{k+1}\right)$ имеет $k$ перемен знака, то $\left.\varepsilon_{n+1, k}(I)=\varepsilon_{n k}\left(I \backslash j_{1}\right), 2\right)$ если для $j_{1}<j_{2} \in J \in C(m, n+1), a \in\{0,1\}$ имеем равенство $\varepsilon_{n k}(J)=(-1)^{a} \varepsilon_{n-2, k-2}\left(J \backslash\left\{j_{1}, j_{2}\right\}\right)$, то не выполняется хотя бы одно из условий $\varepsilon_{n-1, k-1}\left(J \backslash j_{1}\right)=(-1)^{a+1} \varepsilon_{n-1, k-1}\left(J \backslash j_{2}\right), \varepsilon_{n, k-1}(J)=(-1)^{a} \varepsilon_{n-2, k-2}\left(J \backslash\left\{j_{1}, j_{2}\right\}\right) . И_{3}$ теоремы 1 вытекает следующее утверждение.

СледСтвиЕ 2. Если для функции $f d_{k}(J) \neq 0$ при всех $k-1 \leqslant n, J \in C(m, n+1)$, mo $\left(\varepsilon_{n k}: J \mapsto \operatorname{sign} d_{k}(J)\right) \in B(m)$.

Ряд Ньютона в теореме 1 получается применением тождества Бине-Коши к произведению $(k \times \infty)$-матрицы Опитца $(f[i, j])[12]$ и $(\infty \times k)$-матрицы $\left(\left(y-x_{1}\right) \cdots\left(y-x_{j-1}\right)\right)$ (утверждение 2) получается сравнением 1) с общим рядом Ньютона [2]). Применяя к матрице Опитца тождество Турнбулла [13], получим соотношения для коэффициентов ряда Ньютона (мы ограничились частньп случаем 3 )), и затем можно аналогично перейти к комбинаторным аксиомам для в.п.Б.

Для набора $F$ вещественных функций $m$ переменных обозначим через $S(F)$ группу перестановок, действующих тождественно на $F$. Назовем в.п.Б. $B\left(S_{m}, F\right)$ множество классов эквивалентности линейных порядков на $S_{m} / S(F)$, для каждого из которых при любых фиксированных $g \in S_{m},(i j) \notin g S(F) g^{-1}$ одинаков порядок на всех парах $(s g S(F), s(i j) g S(F))_{s}, s \in$ $S\left(\partial_{i j} g(F)\right)\left((A, B)_{s}=(A, B)\right.$, если $s(i)<s(j)$, и $(A, B)_{s}=(B, A)$, если $\left.s(i)>s(j)\right)$. Два порядка эквивалентны, если они отличаются в паре рядом стоящих элементов, не связанных предыдущим условием (частичный порядок на $B\left(S_{m}, F\right)$ вводится аналогично [4]). Примеры: если $F$ - множество кососимметрических функций, то $S(F)$ - группа четных перестановок $A_{m}$ и $B\left(S_{m}, F\right)$ состоит из двух порядков $A_{m}<S_{m} \backslash A_{m}, S_{m} \backslash A_{m}<A_{m}$; можно показать, что $B\left(S_{m}, \partial_{n-1} \cdots \partial_{1} F_{1, m-1}\right)=B(m, n)$, где $F_{1, m-1}$ - множество всех функций, симметричных по последним $m-1$ переменным.

Назовем $f \in F$ общей, если $\partial_{i j} g\left(f\left(x_{1}, \ldots, x_{m}\right)\right) \neq 0$ для всех $g \in S_{m},(i j) \notin g S(F) g^{-1}$. Упорядочивая смежные классы $h S(F)$ по величине $h\left(f\left(x_{1}, \ldots, x_{m}\right)\right)$, для общей $f$ получим элемент $B\left(S_{m}, F\right)$. Поскольку любая композиция $\partial$ э.р.p. является линейной комбинацией перестановок, то последовательность чисел $h \partial\left(f\left(x_{1}, \ldots, x_{m}\right)\right)$ можно представить как последовательность значений линейной функции $\sum g\left(f\left(x_{1}, \ldots, x_{m}\right)\right) t_{g}$.

Функции можно исключить из определения $B\left(S_{m}, F\right)$, поскольку оно зависит только от групп симметрий. Отметим два направления для обобщений: если $x_{i}=x_{j}$, то $\partial_{i j}\left(f\left(x_{i}\right)\right)=f^{\prime}\left(x_{i}\right)$ (для $B(m, n)$ см. [14]); э.p.p. можно определить для любой группы, порожденной отражениями: если $r$ - отражение, $(\cdot, \cdot)$ - инвариантное скалярное произведение, то $\partial_{r}(f(x))=(f(x)-$ $f(r(x))) /(x, a)$, где $a$ - отвечающий $r$ корень.

\section{СПИСОК ЛИТЕРАТУРЫ}

[1] Г. Г. Ильюта // Докл. РАН. 1996. Т. 347. № 3. С. 306-308. [2] A. Lascoux. Notes on interpolation in one and several variables // http://phalanstere.univ-mlv.fr/ al. [3] G. Ziegler // Topology. 1993. V. 32. № 2. P. 259-279. [4] Yu. Manin, V. Schechtman // Adv. Stud. Pure Math. 1989. V. 17. P. 289-308. [5] A. Vince // J. Algebraic Combin. 2000. V. 11. № 2. P. 155-178. [6] W. Chen, J. Louck // Adv. Math. 1996. V. 117. №1. P. 147-156. [7] A. Lascoux, P. Pragacz // J. Symbolic Comput. 2003. V. 35. №6. P. 689-710. [8] H. Salzer // Z. Angew. Math. Mech. 1984. V. 64. P. 45-50. [9] G. Claessens // Aequationes Math. 1979. V. 19. P. 104-112. [10] J. Tan // J. Comput. Math. 2001. V. 19. №4. P. 433-444. [11] Г. Г. Ильюта // Изв. PAH. Cер. матем. 2003. T. 67. № 5. C. 3-34. [12] G. Opitz // Z. Angew. Math. Mech. 1964. V. 44. Sonderheft. P. T52-T54. [13] B. Leclerc // Adv. Math. 1993. V. 100. № 1. P. 101-132. [14] Г. Г. Ильюта // Функц. анализ и его прил. 1998. Т. 32. № 3. С. 76-79.

Московский государственньй открытьй педагогический университет
Представлено С.М. Гусейн-Заде Принято редколлегией 25.01.2005 